\title{
"Comparative Evaluation Of Biofilm Development Of Candida Albicans On Abraded Surfaces Of Heat Cure PMMA And Flexi Denture Material": An In Vitro Study
}

\author{
Raghunath Patil ${ }^{1}$, Heena Sharma ${ }^{2}$, Dolly Gupta ${ }^{3}$ \\ Department of Prosthodontics including Crown and bridge, K.L.E. V.K. Institute of Dental Sciences, Belagavi, \\ Karnataka
}

\begin{abstract}
:
Purpose: Study was to compare the biofilm development of candida albicans on two different types of abraded surfaces of heat cure PMMA and flexi denture material.

Materials And Methods: 4 blocks of each heat cure resin and flexible denture material were prepared of $10 * 10 * 10 \mathrm{~mm}$ and $10 * 10 * 3 \mathrm{~mm}$ in size respectively. All blocks were then subjected to abrasion with p100 grit size dry sand paper or with denture cleansing brush. One colony of Candida albicans GDH 2346 (NCYC 1467) cultured on Sabouraud dextrose agar (SBA) for 48 hrs and was used to inoculate $10 \mathrm{~mL}$ of Peptone water and sugar broth with samples at $37^{\circ} \mathrm{C}$ for 24 hrs. Followed by which gram staining was performed and semiquantification was done after inoculating on SBA for 18 hours at $37^{\circ} \mathrm{C}$.
\end{abstract}

Results: The highest mean colony forming unit was recorded in heat cure PMMA, and lowest mean colony forming unit was recorded in flexible denture material.

Conclusion: Flexible denture material has lesser biofilm development and candidal count as compared to heat cure acrylic resin.

Keywords: Heat cure PMMA, Flexible denture material, Biofilm, Candida albicans

\section{Introduction}

Candida albicans is a known etiologic agent of chronic erythematous candidosis (denture stomatitis). This inflammatory disorder affects approximately $60 \%$ of denture wearers and causes inflammation of oral mucosa in close contact with the denture. As with natural dentition, dentures provide hard nonshedding surfaces that enable the build-up of plaque biofilms over time. Candida biofilm development on denture acrylic resin begins with adhesion, which can either occur directly to the conditioned surface or via a layer of pre-existing denture plaque. ${ }^{1}$ The surface irregularities increase the probability of bacterial accumulation, and Candida adhesion than smooth surface, surface roughness of prostheses providing niches in which the microorganisms are protected from sheer forces and oral hygiene measures. The surface of the resins used in the construction of the prosthesis can be finished and polished using variety of techniques. ${ }^{2}$

Polymethyl methacrylate (PMMA) has been the most popular material used for denture fabrication since its introduction in 1937. It has several advantages such as an excellent esthetic characteristic, low water sorption and solubility, adequate strength, low toxicity, easy repair, and a simple molding processing technique. Nonetheless, it has some problems such as polymerization shrinkage, weak flexural, lower impact strength. In recent years, nylon polymer has attracted attention as a denture base material. Polyamide resin was proposed as a denture base material. Nylon is a generic name for certain types of thermoplastic polymers belonging to the class known as polyamides. Nylon is a crystalline polymer, whereas PMMA is amorphous and low fatigue resistance. On the other side, it is reported that this material has several problems such as water sorption, surface roughness, bacterial contamination, warpage, color deterioration, and difficulty in polishing. ${ }^{3}$ Valplast (flexible denture material) is a nylon thermoplastic material with unique physical and esthetic properties and excellent retention. Thermoplastic resin in spite of all these benefits has some difficulty to adjust and polish. ${ }^{4}$

The purpose of this study was to compare the biofilm development of Candida albicans on two different types of abraded surfaces of heat cure PMMA and flexi denture material. The null hypothesis was that there would not be any difference in biofilm development of Candida albicans on abraded surfaces of heat cure resin and flexi denture material.

\section{Materials and methods}

4 blocks of each heat cure PMMA and flexible denture material were prepared of $10 * 10 * 10 \mathrm{~mm}$ and $10 * 10 * 3 \mathrm{~mm}$ in size respectively (Fig.1). A block was first made in modelling wax of exact dimensions mentioned above and then other 3 blocks were duplicated using the elastomeric rubber based material index of the first disc. 


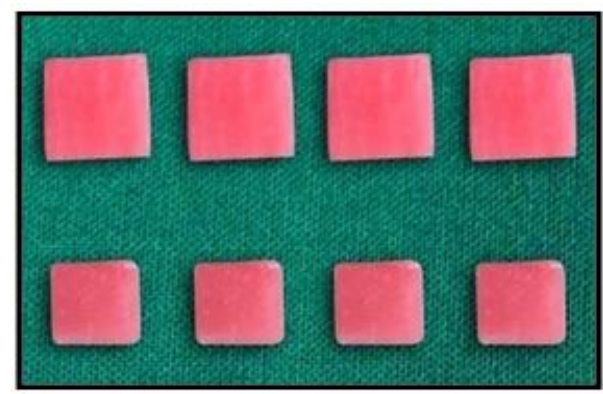

Figure 1.Showing the shape and size of specimens

For Heat cure resin, all 4 blocks were invested in dental plaster and packed using compression moulding technique with heat cure PMMA (MELIODENT). The conventional technique for flasking curing and deflasking was followed. For Flexible denture material, 4 specimen were prepared using injection moulding technique.

All blocks were then subjected to abrasion. 2 blocks of each heat cure resin and flexible denture material were abraded using Sand paper. These specimens were abraded manually with p100 grit size dry sand paper with 10 strokes in one direction parallel to the edge of a ruler, and Other 2 blocks of each material were abraded with denture cleansing Brush (CLANDEN), 400 strokes in one direction and another 400 strokes in opposite direction. All these abrasion procedure is done by one person. After abrading all the blocks, blocks were sterilsed with microwave (600 watt) for 6 mins. Heat cured resin blocks abraded with sandpaper was named - HS, and the ones abraded with brush was named as HB. Sameway, for flexible denture material FS and FB was named(Fig.2).

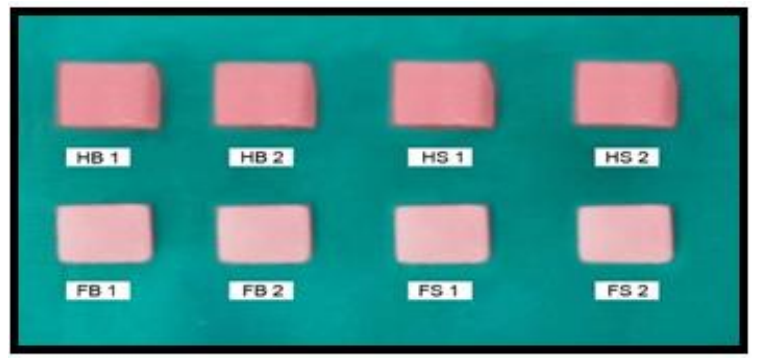

Figure 2. Naming for each specimen

\section{Microbiological testing}

One colony of Candida albicans GDH 2346 (NCYC 1467) cultured on Sabouraud dextrose agar (SBA) for 48 hrs and was used to inoculate $10 \mathrm{~mL}$ of Peptone water and sugar broth with samples at $37^{\circ} \mathrm{C}$ for $24 \mathrm{hrs}$. Test tube named HB contained both HB1 and HB2 samples and HS contained HS1 and HS2. Sameway for flexible denture material samples. After 24 hours samples were checked for biofilm production by taking swabs and performing Gram staining. For semi-quantitation swabs were taken and inoculated on fresh sterile SBA and incubated at $37^{\circ} \mathrm{C}$ (Fig.3)

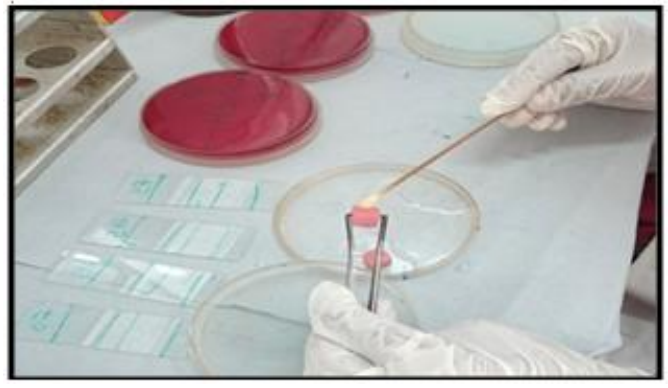

Figure 3. Inoculation of swabs.

They were incubated for 18 hours and then the number of colonies were counted which is then multiplied by 100 to get $\mathrm{CFU} / \mathrm{ml}$ (Fig.4) 

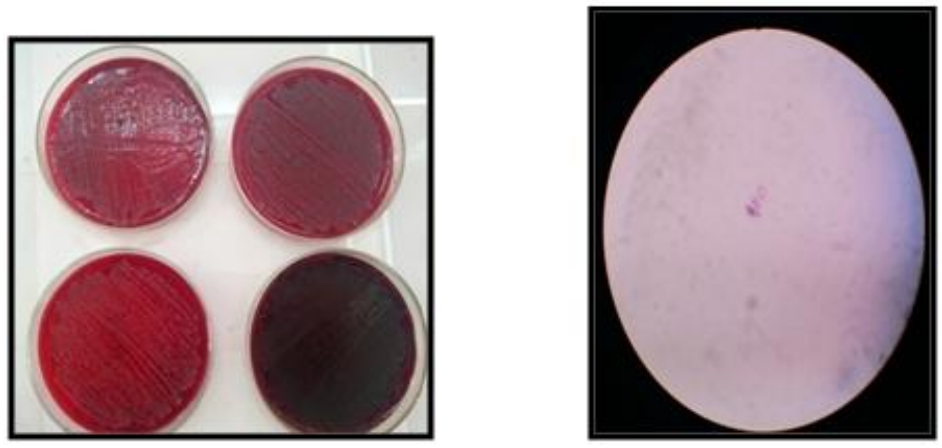

Figure 4. Incubated colonies

Data were presented as mean and standard deviation (SD) values. Unpaired T-Test (Non-parametric) was used for comparison between mean values.

\section{Results}

Candidal count of all the samples is shown in Table 1. Results showed that highest mean colony forming unit was recorded in heat cure acrylic resin, $435000 \mathrm{CFU} / \mathrm{ml}$, and lowest mean colony forming unit was recorded in flexible denture material, $175500 \mathrm{CFU} / \mathrm{ml}$ (Table 2). Comparing the two material, even the sand paper (rougher) abraded flexible denture material has lesser candidal count then brush abraded (smoother) heat cure acrylic resin. The surface roughness play a major role in microbiological attachment, more rougher the surface more the bacterial count.

Table 1. Colony count of the specimens

\begin{tabular}{|l|l|}
\hline \multicolumn{1}{|c|}{ SAMPLES } & $\begin{array}{l}\text { CANDIDAL COUNT } \\
\text { (CFU/ml Unit) }\end{array}$ \\
\hline Heat cure sand paper abraded(HS) & 560000 \\
& 550000 \\
\hline Heat cure brush abraded(HB) & 320000 \\
& 310000 \\
\hline Flexible denture sand paper abraded(FS) & 240000 \\
& 210000 \\
\hline Flexible denture brush abraded(FB) & 110000 \\
& 150000 \\
\hline
\end{tabular}

Table 2. Mean $\pm S D$ values of Colony count of specimens.

\begin{tabular}{|c|c|c|c|c|}
\hline $\begin{array}{c}\text { Sl. } \\
\text { No. }\end{array}$ & Materials & Mean & $\begin{array}{c}\text { Standard } \\
\text { Deviation }\end{array}$ & P value \\
\hline 1 & Heat cured & 435000 & 11860.35 & \multirow{2}{*}{0.021} \\
\hline 2 & Flexi & 177500 & 58523.45 & \\
\hline
\end{tabular}

\section{Discussion}

Adhesion of microorganisms, especially yeasts, to the denture base materials is an important issue that compromises its service and efficacy. ${ }^{3}$ Rougher surfaces contribute to microbial colonization and biofilm formation. Bacterial and fungal species have more of a propensity to adhere to rough denture base materials.one study has suggested that, the conventional polishing technique used for PMMA provided a polyamide surface roughness below the accepted threshold $\mathrm{Ra}$ value. Roughness threshold for PMMA and polyamide was considered appropriate to accept the threshold $\mathrm{Ra}=0.2 \mu \mathrm{m}$ in order to explain the results of this study. ${ }^{5}$

The results of this study supports rejecting the null hypothesis that the there is no statistically significant difference in biofilm development of Candida albicans on abraded surfaces of heat cure resin and flexi denture material. Flexible denture material has less biofilm development even on rough surface (sand paper) as compared to smoother surface (i.e.brush abraded) of heat cure acrylic resin. Candida growth on the polyamide resin was lower than that observed on the PMMA material. The surface roughness play a major role in microbiological attachment, more rougher the surface more the candidal count. ${ }^{7}$ Surface roughness and surface free energy (SFE) may contribute to the positively correlated rate of microbial colonization and plaque maturation on surfaces. ${ }^{8}$

The result of the microbiological study have showed that the finishing and polishing technique that show lower surface roughness value have less number of Candidal cells attachment. This can be explained that 
materials with the roughest surface may serve as reservoir, with surface irregularities providing an increase microorganism retention and protection from shear forces, rough surface has irregularities inducing adhesion of Candida and bacteria, these superficial defect such as voids and micro cracks on surface were possible sites for Candidal adhesion. ${ }^{9}$

\section{Conclusion}

Within the limitations of this in vitro study, the following conclusions were drawn:

1. Flexible denture material has lesser biofilm development and candidal count as compared to heat cure acrylic resin, and

2. Specimen that has lower surface roughness value have less number of Candidal cells attachment

\section{References}

[1]. Jackson S, Coulthwaite L, Loewy Z, Scallan A, Verran J. Biofilm development by blastospores and hyphae of Candida albicans on abraded denture acrylic resin surfaces, J Prosthet Dent 2014;112:988-93

[2]. Mustafa MJ, Amir HM. Evaluation of Candida albicans attachment to flexible denture base material (Valplast) and heat cure acrylic resin using different finishing and polishing techniques. J Bagh College Dentistry 2011; 23(4):36-41

[3]. Mahroo Vojdani, Rashin Giti. Polyamide as a Denture BaseMaterial: A Literature Review J Dent Shiraz UnivMed Sci., 2015 March; 16(1 Suppl): 1-9.

[4]. Michael DiTolla. Valplast Flexible, Esthetic partial dentures.Clinical Techniques \& Procedures 2004;5(1)

[5]. Menaka A. Abuzar, Suman Bellur, Nancy Duong, Billy B. Kim, Priscilla Lu, Nick Palfreyman et al. Evaluating surface roughness of a polyamide denture base material in comparison with poly (methyl methacrylate) Journal of Oral Science, 2010 , Vol. 52, No. 4, 577-581.

[6]. Charman KM, Fernandez P, Loewy Z, Middleton AM. Attachment of Streptococcus oralis on acrylic substrates of varying roughness Lett Appl Microbiol. 2009 Apr;48(4):472-7

[7]. Mieszko Wieckiewicz, Volker Opitz, Gert Richter, and Klaus W. Boening. Physical Properties of Polyamide-12 versus PMMA. Denture Base Material BioMed Research International, Volume 2014 (2014), Article ID 150298, 7 Pages.

[8]. T.D. Morgan and M. Wilson. The effects of surface roughness and type of denture acrylic on biofilm formation by Streptococcus oralis in a constant depth film fermentor. Journal of Applied Microbiology 2001;91:47-53.

[9]. Nevzatoğlu EU, Ozcan M, Kulak-Ozkan Y, Kadir T. Adherence of Candida albicans to denture and silicone based - resilient liner material with different surface finish line. Clin Oral Investig. 2007 Sep;11(3):231-6. 